\title{
Leaders
}

\section{Guidelines for screening for Cryptosporidium in stools: Report of a joint working group ${ }^{\star}$}

\author{
D P Casemore, C Roberts
}

\begin{abstract}
*Members of working group:

Dr Donald Campbell, Environmental Health (Scotland) Unit, representing the Unit and British Society for the Study of Infection.

Dr David Casemore

(Secretary), representing

PHLS.

Dr Peter Chiodini, Hospital for Tropical Diseases,

London.

Dr Anne Dawson,

Department of Health.

Dr James Hutchinson,

BUPA, representing the

Independent Hospitals

Independen

Drsociation.

representing PHLS

Communicable Disease

Surveillance Centre

(CDSC).

Dr Colin Roberts PHLS

HQ, Group Convener

(Chairman).

Dr David Shanson,

Westminster Hospital,

representing the Royal

College of Pathologists and

the Hospital Infection

the Hosiety.

Dociety.

Dr Paul Wright, Royal Eas

Sussex Hospital, Hastings,
representing the Association

of Medical Microbiologists.

PHLS shire, ${ }^{3}$ a group of experts was set up, jointly by the Department of Health and the Department of the Environment, under the chairmanship of Sir John Badenoch. ${ }^{2}$ The remit of the group of experts was to examine, inter alia, the occurrence and extent of cryptosporidium in water supplies, to assess the public health importance, and to assess methods for monitoring supplies. It reviewed and considered the evidence, and, in formulating advice, made several recommendations, including the need to review clinical laboratory diagnosis and surveillance of human cryptosporidiosis. ${ }^{4}$

Recommendation 10 of the Badenoch Report stated that the Public Health Laboratory Service (PHLS), in association with other National Health Service (NHS) and private laboratories, should review and standardise policies for examining faecal samples for cryptosporidium. Such a group was subsequently set up to address this recommendation. The Badenoch Report had identified several deficiencies and problem areas, which were discussed by the Joint Working Group, as described below. The Working Group defined certain key points and then made several recommendations.
\end{abstract}

Cryptosporidium

Reference Unit, Public

Health Laboratory,

Glan Clwyd Hospital,

Bodelwyddan, Rhyl,

Clwyd, LL18 5UJ

D P Casemore

Public Health

Laboratory Service,

London

C Roberts

Correspondence to:

D P Casemore

Accepted for publication

18 May 1992

\section{Introduction}

The protozoan parasite Cryptosporidium sp is now recognised as an important cause of gastrointestinal infection in both immunocompromised and in otherwise healthy subjects. ${ }^{1}$ In recent years the importance of water as a route of transmission has been increasingly recognised. ${ }^{12}$ As a result, and in an urgent response to public concern resulting from an outbreak of cryptosporidiosis in Swindon and Oxford-

\section{Key points}

(1) Clinical diagnostic screening and reporting practices vary greatly between different laboratories. This led to difficulties for the expert group in interpreting the likely importance of changes in figures reported by the PHLS Communicable Diseases Surveillance Centre (CDSC) and by the Communicable Disease
(Scotland) Unit (CD(S)U). Questions were also raised concerning the reliability of identification of outbreaks. In the latter case there may be failure to identify an outbreak because of inadequate screening of faeces, epidemiological investigation may be confused because of variability of screening practice, or "pseudooutbreaks" may be created because of enhanced screening and change of ascertainment, or from misdiagnosis. ${ }^{5}$

Since the first reports of Cryptosporidium $\mathrm{sp}$ to the CDSC in 1983, the number of laboratories within the United Kingdom which screen faeces has increased, but to an unknown extent. In some laboratories, however, screening has been tried and then abandoned, or restricted for logistical or other reasons; in others, whatever the policy, specimen selection criteria may vary according to pressure of work or the enthusiasm of the staff. In some laboratories all diagnostic stool samples are screened, while in others only those from a highly selected group (such as fluid stools from patients younger than 5 years) are screened. The data available do not support the use of stool consistency as a reliable selection criterion. $^{6}$

A two year prospective survey by 16 PHLS laboratories produced data on cryptosporidium and other pathogens in all age groups and included denominator data. ${ }^{7}$ This showed the absolute and relative frequency of identifications of cryptosporidium in that population and provided a basis for a meaningful criterion based on age. Thus the survey indicated that, of the stool samples examined for cryptosporidial oocysts, $60 \%$ of those which were found to be positive occurred in children up to 15 years. Extending the age range to 44 years yielded more than $90 \%$ of the positive results found in that study. ${ }^{67}$

(2) Various screening methods are currently being used, some more reliable or more sensitive than others. Laboratories also vary in the extent to which objective criteria are applied to identification. It is clearly essential, both clinically and in public health control, to be able to rely on accurate identification of organisms implicated in individual cases of disease and in 
outbreaks. It is thus recognised that quality assurance, including participation in an external quality assessment scheme, such as the UK National External Quality Assessment Scheme (NEQAS), is essential. It is also essential to ensure that internal quality control prevents misidentification of oocyst-like bodies. For example, a "pseudo-outbreak" was investigated recently which resulted from a failure of internal laboratory quality control and consequent misidentification of oocyst-like bodies. $^{5}$

(3) It is recognised that laboratory data derived from routine stool examination are subject to many biases. Submission of stools to the laboratory is highly selective and laboratory practice also varies with regard to the range of agents looked for and the methods used. Laboratories also vary in their practice for reporting findings to their local authority, or to $\mathrm{CDSC} / \mathrm{CD}(\mathrm{S}) \mathrm{U}$. With regard to cryptosporidium, it is difficult to compare data among different age groups, areas, etc, or even among different laboratories in the same area, because of these differing selection criteria and the absence of denominator data (that is, the number and categories of the population sampled, including negative findings). The figures reported to $\mathrm{CDSC} / \mathrm{CD}(\mathrm{S}) \mathrm{U}$ therefore, strictly speaking, represent neither incidence nor prevalence figures although they may reflect them. The data are useful for revealing trends in rates of detection, by time, by place, or by age. Their value is increased if data are derived from a stable or standardised selection policy for screening (especially as typing methods become available to distinguish changes in the prevalent subgroup of an organism, such as outbreak strains)

(4) It will take some time before the full effect of reforms in the system of funding of the NHS, set in train by the British government, will become manifest. The users of laboratory services (clinicians, environmental health departments, etc) will now have to pay for individual investigations, the so-called purchaser-provider system of funding. This is likely to have an influence on clinical and microbiological practice, including the submission and examination of stool samples. It is of great importance that the new arrangements do not result in a diminution of specimen numbers with a consequent loss of valuable epidemiological information. It is essential to ensure that legitimate clinical and epidemiological surveillance studies are maintained and where necessary fostered. Such an information database is invaluable, because it enables health care workers to identify as early as possible significant changes in the pattern of cryptosporidiosis, which in turn prompt the early introduction and targeting of appropriate investigative and control procedures, thus making the most effective use of limited resources.

The consequent altered arrangements for the funding of pathology services and the introduction of clinical budgeting is thus of particular importance in the context of submission of such specimens by the clinician. The extent of specimen submission, and the examination by the laboratory, may be limited because of a perceived lack of immediate relevance or benefit to the patient when such examinations are individually costed. Similarly, if charging for secondary examination by reference units, of specimens submitted to them by diagnostic laboratories, is introduced it may well discourage referral with the consequent loss of valuable data and threat to the referral system.

The Joint Working Group thus identified major areas where there were opportunities for improvement and made the following recommendations:

\section{Cryptosporidium Working Group proposed guidelines:}

(1) SPECIMEN SELECTION CRITERIA:

It is recommended that one of the following options be adopted depending on the capabilities of the individual laboratory.

(a) Wherever possible, all diagnostic faecal specimens from symptomatic individuals should be screened for oocysts of Cryptosporidium $\mathrm{sp}$.

(b) Where this is not feasible, the main criterion for selection should be based on age and should include young adults. Thus all diagnostic faecal specimens from patients aged up to and including 44 years should be screened.

(c) As a minimum requirement, all those specimens from children up to and including 15 years should be screened.

(d) Other selection criteria, particularly stool consistency, are unreliable. However, a history of watery offensive stools, contact with a confirmed case or with animals, or a recent history of travel, may indicate the need to examine a specimen otherwise excluded by the age of the patient, if such a criterion is used.

\section{(2) METHODOLOGY FOR DETECTION:}

Methods for detecting oocysts are described in detail in the Association of Clinical Pathologists' Broadsheet Laboratory methods for diagnosing cryptosporidiosis. ${ }^{6}$ Wherever possible, an auramine method should be used for screening. Confirmation of putative positives should be carried out by preparing a fresh smear from the original specimen (to permit rechecking of patient details), and staining by modified Ziehl-Neelsen to demonstrate typical morphology and staining, and to permit measurement, as illustrated in the Broadsheet.

It is essential to ensure adequate quality assurance and the use of both internal and external positive control material. In cases of doubt samples may be referred to one of the laboratories indicated below, offering a reference service, and who will also provide positive control material.

(3) REPORTING:

It is recommended that positive findings should be reported to the local director of public health or consultant in communicable disease control (consultant in public health ( $\mathrm{CD}$ and $\mathrm{EH})$ in Scotland), for investigation by 
the Environmental Health Department, and to CDSC or $\mathrm{CD}(\mathrm{S}) \mathrm{U}$, as is currently done for other potentially foodborne and waterborne pathogens.

\section{Reference facilities:}

PHLS Cryptosporidium Reference Unit, Public Health Laboratory, Glan Clwyd Hospital, Bodelwyddan, Rhyl, Clwyd LL18 5UJ.

Department of Parasitology, Hospital for Tropical Diseases, 4 St Pancras Way, London NW1 OPE.

Scottish Parasite Diagnostic Laboratory, Stobhill General Hospital, Glasgow G21 3UW.
1 Casemore DP. Epidemiological aspects of human cryptosporidiosis. Epidemiol Infect 1990;104:1-28.

2 Anonymous. Cryptospiridium in water supplies. Report of the Group of Experts. Chairman: Sir John Badenoch. London: HMSO, 1990.

3 Richardson AJ, Frankenberg RA, Buck AC, et al. An outbreak of waterborne cryptosporidiosis in Swindon and Oxfordshire. Epidemiol Infect 1991;107:485-95.

4 Anonymous. Report of the Group of Experts on Cryptosporidium in water supplies. Chairman: Sir John Badenoch. Summary, conclusions and recommendations and Government's response. London: HMSO, 1990.

5 ernment's response. London: HMSO, 1990 . asemore DP. A pseudo-outbrea

6 Casemore DP ACP Broadsheet 128: Laboratory methods for diagnosing cryptosporidiosis. FClin Pathol 1991;44:
f 445-51.

7 Palmer SR, Biffin A. Cryptosporidiosis in England and Wales: prevalence and clinical and epidemiological features. Public Health Laboratory Service Study Group. $\mathrm{Br}$ Med f 1990;300:774-7. 